\title{
Trigonometric B-Spline Interpolation
}

Najim Abdullah Ismaeel

University of Garmian -College of Education - Math. Department

\begin{abstract}
:
In present paper

the objective of the choice for studding trigonometric B-spline is made to show it is gives better approximate result or not of the boundary value problems in ordinary differential equations. By applying B-spline procedures to obtain approximate solution of the boundary value problems of ordinary differential equations with trigonometric B-spline, cubic trigonometric B-spline have motivated the solve of boundary value problems with numerical procedures.
\end{abstract}

\section{Keywords:}

Trigonometric B-spline, singular perturbed, second order boundary value problem.

\section{Trigonometric B-Splines: [3]}

Let $\left\{\mathrm{x}_{\mathrm{i}}\right\}$ be a non-decreasing sequence of real numbers such that $\mathrm{x}_{\mathrm{i}+\mathrm{k}}-\mathrm{x}_{\mathrm{i}}<2 \pi$ for all $i$, where $k \geq 1$ is a given integer. The real-valued functions $T_{i, k}$ on $R$ defined by $\mathrm{T}_{\mathrm{i}, \mathrm{k}}(\mathrm{x})=0$ if $\mathrm{x}_{\mathrm{i}+\mathrm{k}}=\mathrm{x}_{\mathrm{i}}$ and $\mathrm{T}_{\mathrm{i}, \mathrm{k}}(\mathrm{x})=\left[\mathrm{x}_{\mathrm{i}}, \mathrm{x}_{\mathrm{i}+1}, \ldots, \mathrm{x}_{\mathrm{i}+\mathrm{k}}\right]_{\mathrm{t}}\left(\sin \frac{y-x}{2}\right)^{k-1}$ if $\mathrm{x}_{\mathrm{i}+\mathrm{k}}>\mathrm{x}_{\mathrm{i}} \cdot$

\section{Definition 2.1 [28]:}

The normalized trigonometric $B$-splines $T_{i, k}$ associated with the knot sequence $\left\{\mathrm{x}_{\mathrm{i}}\right\}$ which gives higher degree trigonometric B-splines, gives by the following iterative formula

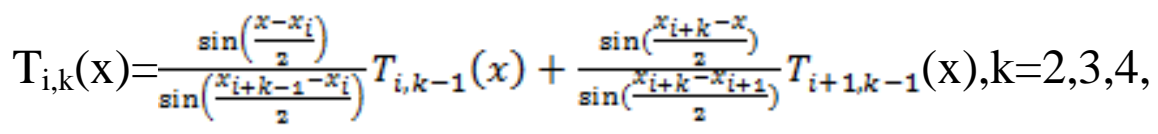


starting with uniform normalized trigonometric B-spline

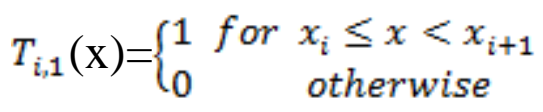

the $\mathrm{T}_{\mathrm{i}, \mathrm{k}}$ functions as defined in (1) has the following properties :

i. Support $\left(\mathrm{T}_{\mathrm{i}, \mathrm{k}}\right)=\left[\mathrm{x}_{\mathrm{i}}, \mathrm{x}_{\mathrm{i}+1}\right)$

ii. $\mathrm{T}_{\mathrm{i}, \mathrm{k}} \geq 0$ for all $\mathrm{x}$ and all $\mathrm{i},($ is positive in the interior of its support and zero otherwise).

iii. $\sum_{i=-\infty}^{\infty} T_{i, k}(\mathrm{x})=1$ for all $\mathrm{x} \in \mathrm{R}$.

Trigonometric B-splines $T_{i, k}$ obtained by applying a linear factor to $T_{i, k-1}$ and $\mathrm{T}_{\mathrm{i}+1, \mathrm{k}-1}$, we see that degree actually increased by 1 at each step .

The spline function $\mathrm{S}(\mathrm{x})$ with respect to the given trigonometric $\quad \mathrm{B}$-spline defined by

$S(x)=\sum_{i=1}^{n} c_{i} T_{i}^{m}(x), c_{i} \in \mathbb{R}, i=1,2, \ldots, n$.

\subsection{Cubic Trigonometric B-spline: $[4,5]$ :}

Let $\pi$ be a uniform partition of the problem domain [a,b] such that $\pi=\left\{a=x_{0}<\right.$ $\left.\mathrm{x}_{1}<\mathrm{x}_{2}<\ldots<\mathrm{x}_{\mathrm{n}-1}<\mathrm{x}_{\mathrm{n}}=\mathrm{b}\right\}$, at the knot points $\mathrm{x}_{\mathrm{i}}, \mathrm{i}=0, \ldots, \mathrm{n}-1, \mathrm{x}_{\mathrm{i}}=\mathrm{x}_{0}+\mathrm{ih}$ and mesh distance $\mathrm{h}=(\mathrm{b}-\mathrm{a}) / \mathrm{n}$, on this partition. The Cubic Trigonometric B-spline defined upon the set of $n+1$ knot points of the problem domain [a, b] as: $\mathrm{TB}_{\mathrm{j}, 3}(x)=\frac{1}{\sigma}\left\{\begin{array}{lr}\sin ^{3}\left(\frac{x-x_{i-2}}{2}\right), & {\left[x_{i-2}, x_{i-1}\right]} \\ \sin ^{2}\left(\frac{x-x_{i-2}}{2}\right) \sin \left(\frac{x_{i}-x}{2}\right)+ & \\ \sin \left(\frac{x-x_{i-2}}{2}\right) \sin \left(\frac{x_{i+1}-x}{2}\right) \sin \left(\frac{x-x_{i-1}}{2}\right) & {\left[x_{i-1}, x_{i}\right]} \\ +\sin ^{2}\left(\frac{x-x_{i-1}}{2}\right) \sin \left(\frac{x_{i+2}-x}{2}\right), & \\ \sin \left(\frac{x-x_{i-2}}{2}\right) \sin ^{2}\left(\frac{x_{i+1}-x}{2}\right)+ & \\ \sin \left(\frac{x-x_{i-1}}{2}\right) \sin \left(\frac{x_{i+1}-x}{2}\right) \sin \left(\frac{x_{i+2}-x}{2}\right) & {\left[x_{i}, x_{i+1}\right]} \\ +\sin \left(\frac{x-x_{i}}{2}\right) \sin ^{2}\left(\frac{x_{i+2}-x}{2}\right), & {\left[x_{i+1}, x_{i+2}\right]} \\ \sin ^{3}\left(\frac{x_{i+2}-x}{2}\right) & \text { otherwise } \\ 0, & \end{array}\right.$ where $\emptyset=\sin (h) \sin \left(\frac{h}{2}\right) \sin \left(\frac{3 h}{2}\right)$ 
It is worth mentioning that $\operatorname{CTB}_{j}(x)$ are twice continuously differentiable piecewise on the problem domain $[a, b]$. Now let $\mathrm{S}(\mathrm{x})$ be the spline interpolating function at the nodal points, then $\mathrm{S}(\mathrm{x})$ can be written as $\mathrm{S}(\mathrm{x})=\sum_{j=-1}^{n+1} c_{j} T B_{j, 3}$.

$\mathrm{S}(\mathrm{x})$ is approximate solution of differential equation where $\mathrm{C}_{\mathrm{j}}$ 's are unknown coefficients, and $T B_{j, 3}(x)$ are cubic trigonometric B-spline functions. To solve boundary value problem of second order with using cubic trigonometric B-spline functions $C T B_{j}$. It required $C T B_{j}, C T B_{j}^{\prime}$, and $C T B_{j}^{\prime \prime}$ been evaluated at the nodal points, that are summarized in the following table 1.

Table 1. The values $B_{i, 3}, B_{i, 3}^{\prime}$ and $B^{\prime \prime}{ }_{i, 3}$

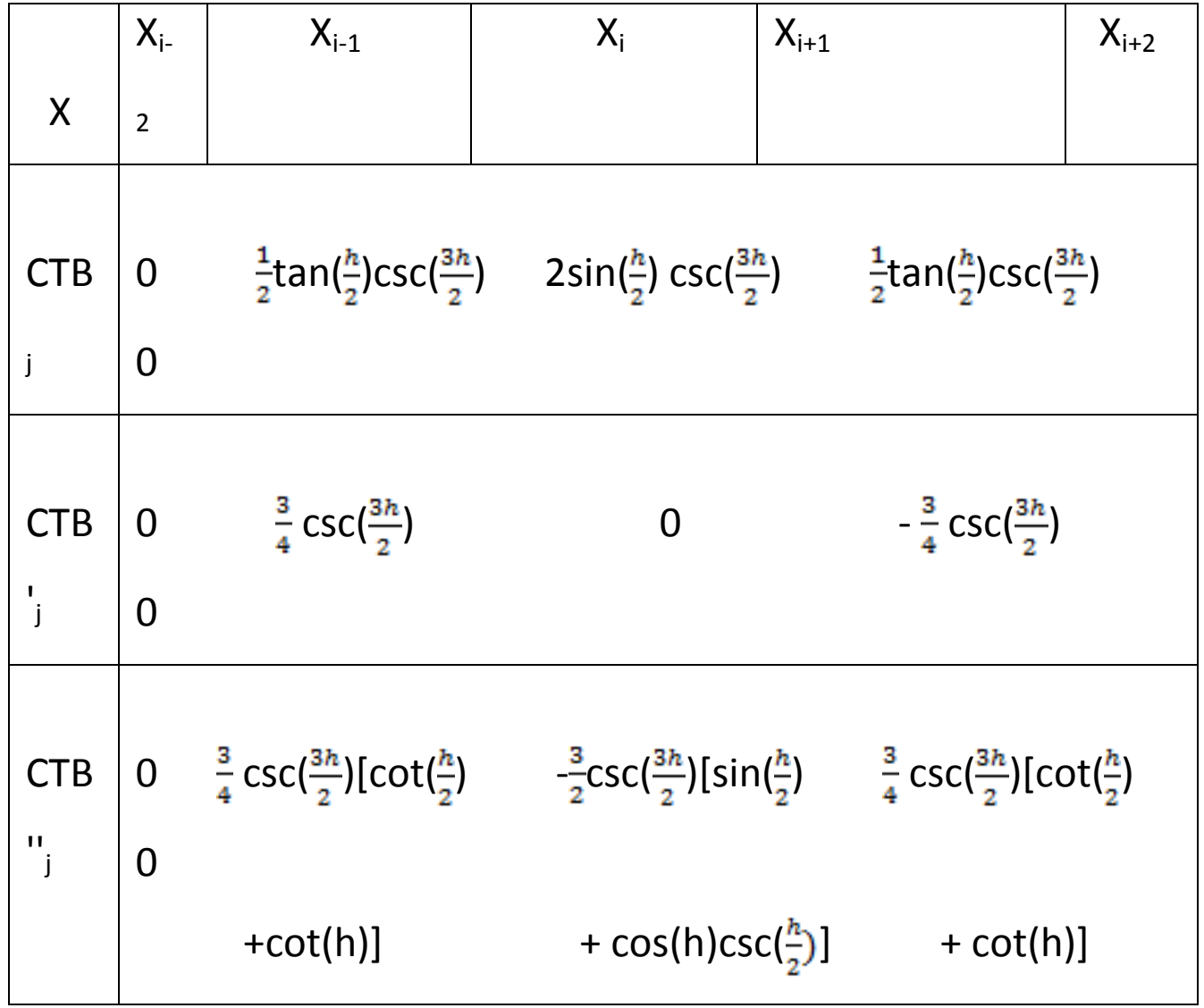

\subsection{Description of the Method:}

Consider the self-adjoint second order singularly perturbed boundary value problem of the form

$$
l u(x)=-\epsilon u^{\prime \prime}(x)+a(x) u(x)=f(x)
$$




$$
u(0)=\alpha
$$

$u(1)=\beta$

where $\alpha$ and $\beta$ are constants and $\in$ is a small positive parameter $(0<E \leq 1), a(x)$ and $\mathrm{f}(\mathrm{x})$ are sufficiently smooth functions. Let $\mathrm{a}(\mathrm{x})=\mathrm{a}=$ constant and let $u(x)=S(x)=\sum_{j=-1}^{n+1} c_{j} T B_{j, 3}$ is approximate solution of (3). Then let $\mathrm{x}_{0}, \mathrm{x}_{1}, \ldots, \mathrm{x}_{\mathrm{n}}$ be $\mathrm{n}+1$ grid points in the interval $[0,1]$. So we have $\mathrm{x}_{1}=\mathrm{x}_{0}+\mathrm{ih}$ where $\mathrm{h}=\mathrm{x}_{\mathrm{i}+1}-\mathrm{x}_{\mathrm{i}}=\frac{1}{n}$ at the knot points, and $\mathrm{x}_{0}=0, \mathrm{x}_{\mathrm{n}}=1, \mathrm{i}=1,2, \ldots, \mathrm{n}$, we get:

$S\left(x_{i}\right)=\sum_{j=-1}^{n+1} c_{j} T B_{j, 3}\left(x_{i}\right)$

$S^{\prime}\left(x_{i}\right)=\sum_{j=-1}^{n+1} c_{j} T B_{j, 3}^{\prime}\left(x_{i}\right)$

$S^{\prime \prime}\left(x_{i}\right)=\sum_{j=-1}^{n+1} c_{j} T B_{j, 3}^{\prime \prime}\left(x_{i}\right)$

substituting the value of equations (5) and (7) in equation (3) we get:

$-\in \sum_{j=-1}^{n+1} c_{j} T B_{j, 3}^{\prime \prime}\left(x_{i}\right)+a\left(x_{i}\right) \sum_{j=-1}^{n+1} c_{j} T B_{j, 3}\left(x_{i}\right)=f\left(x_{i}\right), i=0,1,2, \ldots, n$

and the boundary condition becomes,

$\sum_{j=-1}^{n+1} c_{j} T B_{j, 3}\left(x_{0}\right)=\alpha$

$\sum_{j=-1}^{n+1} c_{j} T B_{j, 3}\left(x_{n}\right)=\beta$

the values of the spline function at the knot points are determined by using table (1), and substituting in (8)-(10), we get a system of $(n+3) \times(n+3)$ equations with $(n+3)$ unknown. Now we write the above system of equations in the following form

$\mathrm{SX}_{\mathrm{n}}=\mathrm{I}_{\mathrm{n}}$

where $X_{n}=\left(c_{-1}, c_{0}, \ldots, c_{n+1}\right)^{T}$ are unknowns

$\mathrm{I}_{\mathrm{n}}=\left(\alpha, \gamma, f\left(x_{0}\right), \ldots, f\left(x_{n}\right), \beta\right)^{T}$ 
$\left(\begin{array}{lr}\sin ^{3}\left(\frac{x-x_{i-2}}{2}\right), & {\left[x_{i-2}, x_{i-1}\right]} \\ \sin ^{2}\left(\frac{x-x_{i-2}}{2}\right) \sin \left(\frac{x_{i}-x}{2}\right)+ & \\ \sin \left(\frac{x-x_{i-2}}{2}\right) \sin \left(\frac{x_{i+1}-x}{2}\right) \sin \left(\frac{x-x_{i-1}}{2}\right) & \\ +\sin ^{2}\left(\frac{x-x_{i-1}}{2}\right) \sin \left(\frac{x_{i+2}-x}{2}\right), & {\left[x_{i-1}, x_{i}\right]}\end{array}\right.$

Since $\quad \mathrm{TB}_{\mathrm{j}, 3}(\mathrm{X})=\frac{1}{\emptyset}\left\{\sin \left(\frac{x-x_{i-2}}{2}\right) \sin ^{2}\left(\frac{x_{i+1}-x}{2}\right)+\right.$

$$
\begin{array}{lr}
\sin \left(\frac{x-x_{i-1}}{2}\right) \sin \left(\frac{x_{i+1}-x}{2}\right) \sin \left(\frac{x_{i+2}-x}{2}\right) & \\
+\sin \left(\frac{x-x_{i}}{2}\right) \sin ^{2}\left(\frac{x_{i+2}-x}{2}\right), & {\left[x_{i}, x_{i+1}\right]} \\
\sin ^{3}\left(\frac{x_{i+2}-x}{2}\right) & {\left[x_{i+1}, x_{i+2}\right]} \\
0, & \text { otherwise }
\end{array}
$$

$\mathrm{TB}_{-1,3}(\mathrm{X})=\frac{1}{\emptyset}\left\{\begin{array}{l}\sin ^{3}\left(\frac{x_{1}-x}{2}\right), \\ 0,\end{array}\right.$

$\mathrm{TB}_{0,3}(\mathrm{X})=\frac{1}{\emptyset}\left\{\begin{array}{l}\sin \left(\frac{x-x_{0}+2 h}{2}\right) \sin ^{2}\left(\frac{x_{1}-x}{2}\right)+ \\ \sin \left(\frac{x-x_{0}+h}{2}\right) \sin \left(\frac{x_{1}-x}{2}\right) \sin \left(\frac{x_{2}-x}{2}\right) \\ +\sin \left(\frac{x-x_{0}}{h}\right) \sin ^{2}\left(\frac{x_{2}-x}{2}\right) \\ \sin ^{3}\left(\frac{x_{2}-x}{2}\right) \\ 0,\end{array}\right.$

$\mathrm{TB}_{1,3}(\mathrm{X})=\frac{1}{\emptyset}\left\{\begin{array}{l}\sin ^{3}\left(\frac{x-x_{0}+h}{2}\right), \\ \sin \left(\frac{x-x_{0}+h}{2}\right) \sin ^{2}\left(\frac{x_{2}-x}{2}\right)+ \\ \sin \left(\frac{x-x_{0}}{2}\right) \sin \left(\frac{x_{2}-x}{2}\right) \sin \left(\frac{x_{\mathrm{g}}-x}{2}\right) \\ +\sin \left(\frac{x-x_{1}}{2}\right) \sin ^{2}\left(\frac{x_{\mathrm{g}}-x}{2}\right), \\ \sin ^{3}\left(\frac{x_{\mathrm{z}}-x}{2}\right), \\ 0\end{array}\right.$

$\mathrm{T}^{3} \mathrm{~B}_{\mathrm{n}-1}(\mathrm{X})=\frac{1}{\emptyset}\left\{\begin{array}{l}\sin ^{3} \frac{\left(x-x_{n-\mathrm{a}}\right)}{2} \\ \sin \frac{\left(x-x_{n-\mathrm{g}}\right)}{2} \sin \frac{\left(x_{n-1}-x\right)}{2}+ \\ \sin \frac{\left(x-x_{n-\mathrm{g}}\right)}{2} \sin \frac{\left(x_{n-x}\right)}{2} \sin \frac{\left(x-x_{n-2}\right)}{2} \\ +\sin ^{2} \frac{\left(x-x_{n-2}\right)}{2} \sin \frac{\left(x_{n+1}-x\right)}{2} \\ \sin ^{3} \frac{\left(x_{n+1}-x\right)}{2} \\ 0\end{array}\right.$ $\left[x_{0}, x_{1}\right]$

otherwise

$$
\begin{aligned}
& {\left[x_{0}, x_{1}\right]} \\
& {\left[x_{1}, x_{2}\right]}
\end{aligned}
$$

otherwise

$$
\left[x_{0}-h, x_{0}\right]
$$

otherwise

$\left[x_{n-3}, x_{n-2}\right]$

$\left[x_{n-2}, x_{n-1}\right]$

$\left[x_{n-1}, x_{n}\right]$

otherwise 


$$
\mathrm{T}^{3} \mathrm{~B}_{\mathrm{n}}(\mathrm{x})=\frac{1}{\emptyset}\left\{\begin{array}{l}
\sin ^{3} \frac{\left(x-x_{n-2}\right)}{2} \\
\sin ^{2} \frac{\left(x-x_{n-2}\right)}{2} \sin \frac{\left(x_{n}-x\right)}{2}+ \\
\sin \frac{\left(x-x_{n-2}\right)}{2} \sin \frac{\left(x_{n}+h-x\right)}{2} \sin \frac{\left(x-x_{n-1}\right)}{2} \\
+\sin ^{2} \frac{\left(x-x_{n-1}\right)}{2} \sin \frac{\left(x_{n+2 h-x}\right)}{2} \\
0
\end{array}\right.
$$

$\mathrm{TB}_{\mathrm{n}+1,3}(\mathrm{X})=\frac{1}{\emptyset}\left\{\begin{array}{l}\sin ^{3}\left(\frac{x-x_{n-1}}{2}\right), \\ 0,\end{array}\right.$

$$
\left[x_{n-2}, x_{n-1}\right]
$$

$$
\left[x_{n-1}, x_{n}\right]
$$

otherwise $\left[x_{n-1}, x_{n}\right]$

otherwise

Form the boundary conditions equations (9)-(10)) we get :

Since $\sum_{j=-1}^{n+1} C_{j} T^{3} B_{j}\left(x_{0}\right)=\alpha$, then

$\frac{1}{2} C_{-1} \tan \left(\frac{h}{2}\right) \csc \left(\frac{3 h}{2}\right)+2 C_{0} \sin \left(\frac{h}{2}\right) \csc \left(\frac{3 h}{2}\right)+\frac{1}{2} C_{1} \tan \left(\frac{h}{2}\right) \csc \left(\frac{3 h}{2}\right)=\alpha$

and since $\sum_{j=-1}^{n+1} C_{j} T^{3} B_{j}\left(x_{n}\right)=\beta$, then

$\frac{1}{2} C_{n-1} \tan \left(\frac{h}{2}\right) \csc \left(\frac{3 h}{2}\right)+2 C_{n} \sin \left(\frac{h}{2}\right) \csc \left(\frac{3 h}{2}\right)+\frac{1}{2} C_{n+1} \tan \left(\frac{h}{2}\right) \csc \left(\frac{3 h}{2}\right)=\beta$

Also

$-\in \sum_{j=-1}^{n+1} C_{j} B_{j}^{\prime \prime}\left(x_{i}\right)+a\left(x_{i}\right) \sum_{j=-1}^{n+1} C_{j} B_{j}\left(x_{i}\right)=f\left(x_{i}\right)$

$\mathrm{i}=0$

$\in\left(\frac{3}{4} C_{-1} \csc \left(\frac{3 h}{2}\right)\left[\cot \frac{h}{2}+\cot (h)\right]+\frac{3}{4} C_{0} \csc \left(\frac{3 h}{2}\right)\left[\cot \frac{h}{2}+\cot (h)\right]+\frac{3}{4} C_{1} \csc \left(\frac{3 h}{2}\right)\left[\cot \frac{h}{2}+\right.\right.$ $\cot (h)])+a\left(x_{0}\right)\left(\frac{1}{2} c_{-1} \tan \left(\frac{h}{2}\right) \csc \left(\frac{3 h}{2}\right)+2 c_{0} \sin \left(\frac{h}{2}\right) \csc \left(\frac{3 h}{2}\right)+\frac{1}{2} c_{1} \tan \left(\frac{h}{2}\right) \csc \left(\frac{3 h}{2}\right)\right)=$ $f\left(x_{0}\right)$

$\mathrm{i}=1$

$\in\left(\frac{3}{4} C_{0} \csc \left(\frac{3 h}{2}\right)\left[\cot \frac{h}{2}+\cot (h)\right]-\frac{3}{2} \csc \left(\frac{3 h}{2}\right)\left[\sin \left(\frac{h}{2}\right)+\cos (h) \csc \left(\frac{h}{2}\right)\right]\right)+$ $a\left(x_{1}\right)\left(\frac{1}{2} C_{0} \tan \left(\frac{h}{2}\right) \csc \left(\frac{3 h}{2}\right)\right.$

$\left.+2 C_{1} \sin \left(\frac{h}{2}\right) \csc \left(\frac{3 h}{2}\right)\right)=f\left(x_{1}\right)$

$\mathrm{i}=2$ 
$-\in\left(\frac{3}{4} C_{1} \csc \left(\frac{3 h}{2}\right)\left[\cot \frac{h}{2}+\cot (h)\right]\right)+a\left(x_{2}\right)\left(\frac{1}{2} C_{1} \tan \left(\frac{h}{2}\right) \csc \left(\frac{3 h}{2}\right)\right)=f\left(x_{2}\right)$

and $\mathrm{i}=\mathrm{n}-2$, then

$$
\begin{aligned}
& -\in\left(\frac{3}{4} C_{n-1} \csc \left(\frac{3 h}{2}\right)\left[\cot \frac{h}{2}+\cot (h)\right]\right)+a\left(x_{n-2}\right) \\
& \left(\frac{1}{2} C_{n-1} \tan \left(\frac{h}{2}\right) \csc \left(\frac{3 h}{2}\right)\right)=f\left(x_{n-2}\right)
\end{aligned}
$$

$\mathrm{i}=\mathrm{n}-1$

$$
\begin{aligned}
& -\in\left(-\frac{3}{2} C_{n-1} \csc \left(\frac{3 h}{2}\right)\left[\sin \left(\frac{h}{2}\right)+\cos (h) \csc \left(\frac{h}{2}\right)\right]+\right. \\
& \left.\frac{3}{4} C_{n} \csc \left(\frac{3 h}{2}\right)\left[\cot \frac{h}{2}+\cot (h)\right]\right)+a\left(x_{n-1}\right) \\
& \left(2 C_{n-1} \sin \left(\frac{h}{2}\right) \csc \left(\frac{3 h}{2}\right)+\frac{1}{2} C_{0} \tan \left(\frac{h}{2}\right) \csc \left(\frac{3 h}{2}\right)\right)=f\left(x_{n-1}\right)
\end{aligned}
$$

$\mathrm{i}=\mathrm{n}$,

$-\in\left(\frac{3}{4} C_{n-1} \csc \left(\frac{3 h}{2}\right)\left[\cot \frac{h}{2}+\cot (h)\right]-\frac{3}{2} \csc \left(\frac{3 h}{2}\right)\left[\sin \left(\frac{h}{2}\right)+\cos (h) \csc \left(\frac{h}{2}\right)\right]+\right.$ $\left.\frac{3}{4} C_{n+1} \csc \left(\frac{3 h}{2}\right)\left[\cot \left(\frac{h}{2}\right)+\cot (h)\right]\right)+a\left(x_{n}\right)\left(\frac{1}{2} C_{n-1} \tan \left(\frac{h}{2}\right) \csc \left(\frac{3 h}{2}\right)+\right.$ $\left.2 C_{n} \sin \left(\frac{h}{2}\right) \csc \left(\frac{3 h}{2}\right)+\frac{1}{2} C_{n+1} \tan \left(\frac{h}{2}\right) \csc \left(\frac{3 h}{2}\right)\right)=f\left(x_{n}\right)$

From equation(11)-(18), so the coefficient matrix is given by

$\left[\begin{array}{ccc}\mathrm{U} & V & \mathrm{U} \\ -\frac{3}{4} \csc \left(\frac{3 h}{2}\right) & 0 & \frac{3}{4} \csc \left(\frac{3 h}{2}\right) \\ S_{0} & T_{0} & S_{0} \\ 0 & S_{1} & T_{1} \\ 0 & & S_{2} \\ 0 & \cdots & \cdots \\ 0 & \ldots & \ldots\end{array}\right.$




$\left.\begin{array}{cccc}0 & 0 & 0 & 0 \\ \vdots & \vdots & \vdots & \vdots \\ 0 & S_{n-1} & 0 & 0 \\ \vdots & T_{n-1} & S_{n-1} & 0 \\ \vdots & S_{n} & T_{n} & S_{n} \\ 0 & U & \mathrm{~V} & \mathrm{U}\end{array}\right]\left[\begin{array}{c}C_{-1} \\ C_{0} \\ \vdots \\ \vdots \\ C_{n-1} \\ C_{n} \\ C_{n+1}\end{array}\right]=\left[\begin{array}{c}\alpha \\ f\left(x_{0}\right) \\ f\left(x_{1}\right) \\ \vdots \\ \vdots \\ f\left(x_{n}\right) \\ \beta\end{array}\right]$

where

$S_{i}=-\frac{3}{4} \in \csc \left(\frac{3 h}{2}\right)\left[\cot \left(\frac{h}{2}\right)+\cot (h)\right]+\frac{1}{2} a\left(x_{i}\right) \tan \left(\frac{h}{2}\right) \csc \left(\frac{3 h}{2}\right)$

$T_{i}=\frac{3}{2} \in \csc \left(\frac{3 h}{2}\right)\left[\sin \left(\frac{h}{2}\right)+\cos (h) \csc \left(\frac{h}{2}\right)\right]+2 a\left(x_{i}\right) \sin \left(\frac{h}{2}\right) \csc \left(\frac{3 h}{2}\right)$

$\left.U=\frac{1}{2} \tan \left(\frac{h}{2}\right) \csc \frac{3 h}{2}\right)$

$V=2 \sin \left(\frac{h}{2}\right) \csc \left(\frac{3 h}{2}\right)$ for $\mathrm{i}=0,1,2, \mathrm{n}-2, \mathrm{n}-1, \mathrm{n}$

\section{Numerical Results}

In this section the purpose is the test of the new method for solving ordinary differential equations of boundary value problems through the following example.

\section{Example:}

Consider the following second order boundary value problem subject to boundary conditions:

$$
-\epsilon y^{\prime \prime}+4 y=\frac{(0.3 x)^{10}}{625},
$$

and boundary conditions $\quad \mathrm{y}(0)=0, \mathrm{y}(1)=0$

\begin{tabular}{|l|l|l|l|}
\hline $\mathrm{N}$ & $10^{-1}$ & $10^{-2}$ & $10^{-3}$ \\
\hline 10 & $6.648297183 \times 10^{-10}$ & $1.994628381 \times 10^{-8}$ & $4.199774960 \times 10^{-9}$ \\
\hline 20 & $4.180808855 \times 10^{-10}$ & $3.324979841 \times 10^{-9}$ & $1.953986258 \times 10^{-5}$ \\
\hline 40 & $3.000763563 \times 10^{-10}$ & $1.439594520 \times 10^{-9}$ & $2.109720459 \times 10^{-8}$ \\
\hline
\end{tabular}




\section{Conclusion:}

In present chapter we used new method to solve boundary value problem by using of cubic trigonometric B-spline interpolation it seems that the absolute errors are small enough and acceptable; consequently the results were convincing. Moreover, we found that use TBS interpolation, gives more accurate results in comparison with use of B-spline interpolation.

\section{Reference}

[1] Athanassion Nikolis, "Numerical Solution of ordinary differential Equations with Quadratic Trigonometric Splines", E-Notes, 4, pp.142-149, (2004).

[2] Dă̆ I., O. Ersoy and ö. Kaçmaz, Eskişehir Osmangozi university, "The Trigonometric cubic B-spline Algorithm for Burgers' Equation", 22 July (2014).

[3] Lyche T.and R. Winther, "A stable Recurrence Relation for Trigonometric BSplines", J. Approx. Theory 25, pp. 266-279, (1979)

[4] Nikolis A.,"'Numerical solution of ordinary differential equation with quadratic trigonometric splines", Applied Mathematics E-Notes, Vol. 4 pp. 142-149, (1995).

[5] Nikolis A. and I. Seimenis, "Solving dynamical Systems with Cubic Trigonometric Splines", Applied Mathematics E-notes, Vol. 5, pp. 116-123, (2005).

[6] Per Erik Koch, Tom Lyche, Marian Neamtu and Larry L. Schumaker, "Control Curve and Knot Insertion for Trigonometric Splines", Advances in Computational Mathematics, pp. 405-424, (1995).

[7] Schoenberg, I.J., "Trigonometric Spline Interpolation", J. Math. Mech, Vol. 13, pp. 795-825, (1964).

[8] Tom Lyche, Larry L. Schumaker and Sonya Stanley "Quasi-Interpolation Based on Trigonometric Spline", (1992). 